\title{
A MONOENERGETIC-SOURCE SOLUTION OF THE STEADY-STATE COSMIC-RAY EQUATION OF TRANSPORT
}

\author{
G. M. WEBB
}

(Received 2 February 1977)

(Revised 12 July 1977)

\begin{abstract}
A monoenergetic point-source solution of the steady-state cosmic-ray equation of transport for cosmic-rays in the interplanetary region in which monoenergetic particles are released isotropically and continuously from a fixed heliocentric position is derived by Lie Theory. A spherically-symmetric model of the propagation region is assumed incorporating anisotropic diffusion with a diffusion tensor symmetric about the radial direction, and the solar wind velocity is radial and of constant speed $V$. Because of the point release the solution is non-spherically-symmetric.
\end{abstract}

\section{Introduction}

The equation of transport for cosmic-rays in the interplanetary region, including convection diffusion and energy changes, was obtained initially by Parker [18], and later by Gleeson and Axford [9], and by Dolginov and Toptygin [5, 6].

Steady-state analytic solutions of the equation have been given by Parker [18, 19], Dolginov and Toptygin [5, 6], Fisk and Axford [8], Toptygin [21], Webb and Gleeson [25, 26], Gleeson and Webb [13] and Webb [23, 24], and numerical solutions have been obtained following Fisk [7] and Urch [22]. In addition, two useful approximate analytic solutions have been obtained by Gleeson and Axford $[10,11]$ and improved upon by Gleeson and Urch [12].

Probably the most fundamental analytic solution at present is the monoenergeticsource or Green's function solution given by Webb and Gleeson [25], without proof, and also by Toptygin [21], who derived it by a Laplace transform method. In this solution monoenergetic particles are released isotropically and at a steady 
rate from a spherical shell at a fixed heliocentric radius, and the resultant redistribution of particles in momentum $p$, is determined as a function of heliocentric radius $r$. The solution was obtained for a model of the interplanetary region in which the effective radial diffusion coefficient $\kappa=\kappa_{0}(p) r^{b}$, with $b$ a constant, $\kappa_{0}(p)$ an arbitrary function of $p$, and the solar wind velocity was assumed radial and of constant speed $V$.

In this paper, a monoenergetic point-source solution of the equation of transport is derived by using Lie Theory (Lie [16], Bluman and Cole [3, 4], Bluman [2], Ovsjannikov [17], Harrison and Estabrook [14]) and the results of Webb [24]. The solution is obtained for a spherically-symmetric model of the interplanetary region incorporating anisotropic diffusion, in which monoenergetic particles are released isotropically and at a steady rate from a fixed point within the region. The solution for the momentum average distribution function $F_{0}(r, \theta, \phi, p)$ is a function of particle momentum $p$, heliocentric position $(r, \theta, \phi)$ where $(r, \theta, \phi)$ are spherical polar coordinates centred on the sun with polar axis along the sun's rotation axis.

The interplanetary magnetic field is assumed radial with a diffusion tensor symmetric about the magnetic field and the solar wind velocity is assumed radial and of constant speed $V$.

The point-source solution is obtained by determining the groups of continuous transformations which map the equation of transport with a monoenergetic-source term onto itself.

In Section 2, the steady-state equation of transport is expressed in a separable form, with separation variables $(x, t, \mu, \phi)$, where $x$ is a function of $r$ and $p, t$ is a function of $p$ and $\mu=\cos \theta$.

In Section 3, we use the results of Ovsjannikov [17] to write down the infinitesimal transformations admitted by the equation of transport and we note the auxiliary conditions which arise when a monoenergetic source term is included.

In Section 4, the point-source solution is obtained by using the infinitesimal generators of Section 3, in conjunction with the similarity solutions of Webb [24]. For completeness we give the spherically-symmetric monoenergetic-source solution of Webb and Gleeson [25] and of Toptygin [21] which may also be derived by group methods.

\section{Separation of the cosmic-ray equation of transport}

With a source of monoenergetic particles of momentum $p_{0}$ released at heliocentric position $\mathbf{r}_{\mathbf{0}}$, the steady-state cosmic-ray equation of transport is (Parker [18], Gleeson and Axford [9], Jokipii and Parker [15], Dolginov and Toptygin [5, 6])

$$
\boldsymbol{\nabla} \cdot\left(\mathbf{V} F_{0}-\underline{\kappa} \cdot \nabla F_{0}\right)-\frac{1}{3 p^{2}} \boldsymbol{\nabla} \cdot \mathbf{V} \frac{\partial}{\partial p}\left(p^{3} F_{0}\right)=\frac{N_{s} \delta\left(\mathbf{r}-\mathbf{r}_{0}\right) \delta\left(p-p_{0}\right)}{4 \pi p_{0}^{2}}
$$


where $F_{0}(\mathbf{r}, p)$ is the mean distribution function with respect to momentum $p$ at heliocentric position $\mathbf{r} ; \mathbf{V}$ is the solar wind velocity; $\underline{\kappa}$ the diffusion tensor; and the right-hand side of equation (2.1) is the source term with $N_{s}$ the injection rate.

The interplanetary magnetic field is assumed radial with diffusion coefficients $\kappa_{\downarrow}$ along and $\kappa_{\perp}$ perpendicular to the magnetic field given by

$$
\kappa_{\mathrm{l}}=\kappa_{0}(p) r^{b}, \quad \kappa_{\perp} / \kappa_{\mathrm{l}}=e=\text { constant. }
$$

By introducing new independent variables $(x, t, \mu, \phi)$ where

$$
\begin{aligned}
& x= \begin{cases}2|1-b|^{-1}\left(r p^{3 / 2}\right)^{(1-b) / 2} & \text { if } b \neq 1, \\
-\frac{1}{2} \ln \left(2 r^{2} p^{3}\right) & \text { if } b=1,\end{cases} \\
& t=-3 \int^{p} \kappa_{0}(z) z^{(1-3 b) / 2} d z / 2 V,
\end{aligned}
$$

and $\mu=\cos \theta$, the equation of transport (2.1) may be written as

$$
\begin{aligned}
\frac{\partial^{2} F_{0}}{\partial x^{2}}+\left(\frac{a_{1}}{x}+a_{2}\right) \frac{\partial F_{0}}{\partial x}-\frac{\partial F_{0}}{\partial t}+\left(a_{3}+\frac{a_{4}}{x^{2}}\right) & {\left[\left(1-\mu^{2}\right) \frac{\partial^{2} F_{0}}{\partial \mu^{2}}-2 \mu \frac{\partial F_{0}}{\partial \mu}+\frac{1}{\left(1-\mu^{2}\right)} \frac{\partial^{2} F_{0}}{\partial \phi^{2}}\right] } \\
& =-Z \delta\left(x-x_{0}\right) \delta\left(t-t_{0}\right) \delta\left(\mu-\mu_{0}\right) \delta\left(\phi-\phi_{0}\right),
\end{aligned}
$$

where

(i) if $b \neq 1, \kappa_{\|}=\kappa_{0}(p) r^{b}, \kappa_{\perp} / \kappa_{\natural}=e$, then

$$
\begin{aligned}
n & =(b+1) /(1-b), \quad a_{1}=2 n+1, \quad a_{2}=0, \\
a_{4} & =e(n+1)^{2}, \quad a_{3}=0, \\
Z & =3 N_{s} x_{0} /\left(8 \pi V p_{0}^{3} r_{0}^{2}|n+1|\right)
\end{aligned}
$$

and

(ii) if $b=1$ so that $\kappa_{\|}=\kappa_{0}(p) r, \kappa_{\perp} / \kappa_{\|}=e$,

$$
\begin{aligned}
& a_{1}=a_{4}=0, \quad a_{2}=-2, \quad a_{3}=e, \\
& Z=3 N_{s} /\left(8 \pi V p_{0}^{3} r_{0}^{2}\right) .
\end{aligned}
$$

\section{The group of the transport equation}

Using the notation $\left(x, t, \mu, \phi, F_{0}\right) \equiv\left(x^{1}, x^{2}, x^{3}, x^{4}, u\right)$ we consider the group of continuous infinitesimal transformations

$$
x^{\prime i}=x^{i}+\varepsilon \xi^{i}(\mathbf{x} ; u), \quad u^{\prime}=u+\varepsilon U(\mathbf{x} ; u), \quad i=1(1) 4,
$$

which leave the transport equation (2.5a) invariant. Writing the equation of 
transport as $M[u]=0$, with $M$ the appropriate differential operator, we require

$$
M^{\prime}\left[u^{\prime}\right]-M[u]=\varepsilon \lambda(\mathbf{x}) M[u]+O\left(\varepsilon^{2}\right),
$$

where $M^{\prime}\left[u^{\prime}\right]$ is obtained from $M[u]$ by replacing $(\mathbf{x}, u)$ by $\left(\mathbf{x}^{\prime}, u^{\prime}\right)$, and $\lambda$ is a function of $\mathbf{x}$ only (Ovsjannikov [17], Chapter 6).

The determining equations for the infinitesimal generators $\left(\xi^{i}, U\right), i=1$ (1) 4 , admitted by the transport equation may be obtained from the results of Ovsjannikov ([17], Chapter 6) or alternatively by the technique of Bluman and Cole [3]. We note, for later reference, that the generators $\left(\bar{\xi}^{i}, \bar{U}\right), i=1$ (1) 4 , for the Green's function solution of the transport equation (2.5a) are the subset of the homogeneous equation generators, satisfying the auxiliary conditions

$$
\bar{\xi}^{i}\left(\mathbf{x}_{0}\right)=0, \quad i=1(1) 4,
$$

corresponding to invariance of the source point $\mathbf{x}_{0}$ and

$$
\sum_{i=1}^{4} \frac{\partial \xi_{i}\left(\mathbf{x}_{0}\right)}{\partial x^{i}}+\lambda\left(\mathbf{x}_{0}\right)=0
$$

corresponding to invariance of the source term.

The infinitesimal generators $\left(\xi^{i}, U\right), i=1$ (1) 4 , of (3.1) depend on the radial dependence of the diffusion tensor according as (i) $\kappa_{\pi}=\kappa_{0}(p) r^{b}, b \neq 1$, or (ii) $\kappa_{\|}=\kappa_{0}(p) r$, and these are given below as Case (i) and Case (ii) respectively.

Case (i) $\kappa_{\Downarrow}=\kappa_{0}(p) r^{b}, b \neq 1, \kappa_{\perp} / \kappa_{\sharp}=e \neq 0$ has generators

$$
\begin{aligned}
& \xi^{1}=\gamma x\left(t-t_{s}\right), \quad \xi^{2}=\gamma\left[\left(t-t_{s}\right)^{2}+a_{s}\right], \\
& \xi^{3}=A \sin \theta \sin \theta_{s} \sin \left(\phi_{s}-\phi\right), \\
& \xi^{4}=A\left[\cot \theta \sin \theta_{s} \cos \left(\phi-\phi_{s}\right)-\cos \theta_{s}\right], \\
& U=\sigma u+\Omega(\mathbf{x}), \quad \sigma=\gamma\left[-\frac{1}{4} x^{2}-(n+1) t+\delta\right],
\end{aligned}
$$

where $\Omega(\mathbf{x})$ is an arbitrary solution of the homogeneous transport equation (2.5a) and $\gamma, t_{s}, a_{s}, A, \theta_{s}, \phi_{s}, \delta$ are arbitrary parameters. The function $\lambda(\mathbf{x}) \mathrm{o}$ equation (3.2) is given by

$$
\lambda=\gamma\left[-\frac{1}{4} x^{2}-(n+1) t+\delta\right]-2 \gamma\left(t-t_{s}\right) .
$$

It is of interest to note that the vector field corresponding to the infinitesimal generators (3.4) has the decomposition

$$
\begin{aligned}
\xi^{i} \partial_{x^{i}}+U \partial_{u}=- & A \partial_{\Phi}+\gamma\left(t_{s}^{2}+a_{s}\right) \partial_{l}-\gamma t_{s}\left(x \partial_{x}+2 t \partial_{l}\right) \\
& +\gamma\left[x t \partial_{x}+t^{2} \partial_{l}+u\left(-\frac{1}{4} x^{2}-n t-t\right) \partial_{u}\right]+\gamma \delta u \partial_{u}+\Omega \partial_{u},
\end{aligned}
$$


where $\Phi$ is the azimuthal angle in a coordinate system with polar axis in the direction $\left(\theta_{s}, \phi_{s}\right)$. Thus the parameter $A$ represents a rotation about this polar axis; $\gamma\left(t_{s}^{2}+a_{s}\right)$ corresponds to a translation in $t ; \gamma t_{s}$ and $\gamma \delta$ correspond to stretches.

With a monoenergetic source term in (2.5a) the generators (3.4) satisfy the auxiliary conditions (3.3) so that if $A, \gamma \neq 0$,

$$
t_{s}=t_{0}, \quad a_{s}=0, \quad \theta_{s}=\theta_{0}, \quad \phi_{s}=\phi_{0}, \quad \delta=\frac{1}{4} x_{0}^{2}+(n+1) t_{0} .
$$

The operator $-A \partial_{\Phi}$ in (3.6) and (3.7) show that the monoenergetic point-source solution with source at $\left(r_{0}, \theta_{0}, \phi_{0}\right)$ is invariant under rotations about an axis through $\left(\theta_{0}, \phi_{0}\right)$. Since the solution is independent of the arbitrary parameter $A$, we find (see equations (4.6), (4.7)) that the point-source solution is independent of the azimuthal angle $\Phi$ about $\left(\theta_{0}, \phi_{0}\right)$.

Case (ii) $\kappa_{1}=\kappa_{0}(p) r, \kappa_{\perp} / \kappa_{\Downarrow}=e \neq 0$.

In this case the transport equation admits the operator

$$
\gamma\left[\left(t-t_{s}\right) \partial_{x}+\left(t-\frac{1}{2} x\right) u \partial_{u}\right]+\delta \partial_{t}-A \partial_{\Phi}+k u \partial_{u}+\Omega \partial_{u} ;
$$

and the function $\lambda(\mathbf{x})$ of equation (3.2) is given by

$$
\lambda=\gamma t-\frac{1}{2} \gamma x+k .
$$

Here $\Omega(\mathbf{x})$ is an arbitrary solution of the homogeneous equation of transport; $\gamma, t_{s}, \delta, A, k$ are arbitrary parameters and $-\partial_{\Phi}$ corresponds to an infinitesimal rotation about an axis through $\left(\theta_{s}, \phi_{s}\right)$ as in equation (3.6).

With a monoenergetic source located at $\left(x_{0}, t_{0}, \mu_{0}, \phi_{0}\right)$, conditions (3.3) give

$$
\delta=0, \quad t_{s}=t_{0}, \quad \theta_{s}=\theta_{0}, \quad \phi_{s}=\phi_{0}, \quad k=\gamma\left(\frac{1}{2} x_{0}-t_{0}\right)
$$

as the parameter values in (3.8) for the point-source solution.

Again the remarks following (3.7) apply.

\section{The monoenergetic point-source solution}

The form of the monoenergetic point-source solution depends on the radial dependence of $\kappa_{\mathrm{\eta}}$. We consider the case $\kappa_{\mathrm{q}}=\kappa_{0}(p) r^{b}, b \neq 1, \kappa_{\perp} / \kappa_{\mathrm{q}}=e$, in detail and sketch the derivation for $\kappa_{\mathbb{1}}=\kappa_{0}(p) r$.

Case $(i) \kappa_{1}=\kappa_{0}(p) r^{b}, b \neq 1$.

To obtain the point-source solution one first integrates the group trajectories

$$
\frac{d x^{i}}{\xi^{i}}=\frac{d u}{U}, \quad i=1(1) 4,
$$

to obtain the invariants of the group (Bluman and Cole $[3,4])$. Using angular variables $(\Theta, \Phi)$ relative to an axis through $\left(\theta_{s}, \phi_{s}\right)$ and integrating the trajectories 
for (3.6) with $\Omega=0$, one obtains the invariants $\left(\eta, \mu^{*}, w, \psi\right)$ :

$$
\begin{aligned}
& \eta=\frac{x}{\left[\left(t-t_{8}\right)^{2}+a_{s}\right]^{t}}, \quad \mu^{*}=\cos \Theta, \quad w=\Phi+\frac{A}{\gamma} \int^{T} \frac{d y}{y^{2}+a_{s}}, \\
& \psi=u \exp \left[\frac{1}{4} \eta^{2} T+\frac{1}{2}(n+1) \ln \left(T^{2}+a_{s}\right)-\chi \int \frac{T}{\left.y^{2}+a_{s}\right]},\right.
\end{aligned}
$$

where

$$
T=t-t_{s}, \quad \chi=\delta-(n+1) t_{s} .
$$

From (4.2) the invariant group solutions of (2.5a) are of the form

$$
u=\exp \left[-\frac{1}{4} \eta^{2} T-\frac{1}{2}(n+1) \ln \left(T^{2}+a_{s}\right)+\chi \int^{T} \frac{d y}{y^{2}+a_{s}}\right] f\left(\eta, \mu^{*}, w\right),
$$

where $f$ is an arbitrary function of $\eta, \mu^{*}, w$. Substitution of the solution form (4.4) into the transport equation (2.5a) yields a partial differential equation for $f\left(\eta, \mu^{*}, w\right)$ :

$$
\begin{aligned}
\frac{\partial f}{\partial \eta^{2}}+ & \frac{2 n+1}{\eta} \frac{\partial f}{\partial \eta}+\left(\frac{1}{4} a_{s} \eta^{2}-\chi\right) f-\frac{A}{\gamma} \frac{\partial f}{\partial w} \\
& +\frac{e(n+1)^{2}}{\eta^{2}}\left[\left(1-\mu^{*^{2}}\right) \frac{\partial^{2} f}{\partial \mu^{*^{2}}}-2 \mu^{*} \frac{\partial f}{\partial \mu^{*}}+\frac{1}{1-\mu^{*^{2}}} \frac{\partial^{2} f}{\partial w^{2}}\right]=0 .
\end{aligned}
$$

To obtain the point-source solution we note that the parameter values (3.7) apply. From the uniqueness of the solution it follows that $f\left(\eta, \mu^{*}, w\right)$ must be independent of the arbitrary parameter $A / \gamma$, and hence independent of $w$ (i.e. of $\Phi)$ by (4.2). Upon solving (4.5) for $f$ and using (4.4) we obtain

$$
\begin{array}{r}
u=\exp \left[-\frac{1}{4} \eta^{2} T-(n+1) \ln T-\frac{1}{4} x_{0}^{2} / T\right] \eta^{-n}\left[C I_{\zeta}\left(\frac{1}{2} x_{0} \eta\right)+D K_{\zeta}\left(\frac{1}{2} x_{0} \eta\right)\right] \\
\times\left[c_{l} P_{l}\left(\mu^{*}\right)+d_{l} Q_{l}\left(\mu^{*}\right)\right]
\end{array}
$$

where

$$
\begin{aligned}
\zeta & =\left[n^{2}+e l(l+1)(n+1)^{2}\right]^{\frac{1}{2}}, \quad n=(b+1)(1-b), \\
\eta & =x /\left(t-t_{0}\right), \quad T=t-t_{0}, \\
\mu^{*} & =\cos \theta \cos \theta_{0}+\sin \theta \sin \theta_{0} \cos \left(\phi-\phi_{0}\right)
\end{aligned}
$$

as the form of the point-source solution. Here $I_{\zeta}(z)$ and $K_{\zeta}(z)$ are modified Bessel functions of the first and second kind, and $P_{l}\left(\mu^{*}\right), Q_{l}\left(\mu^{*}\right)$ are standard solutions of Legendre's equation (Abramowitz and Stegun [1], Sections 9.6, 8.1). Note that the similarity form (4.6) has been given previously in Webb ([24], equations (6.4), (6.6)).

Since we require the solution to be finite at heliocentric radii $r \rightarrow 0$, and $F_{0} \rightarrow 0$ as $r \rightarrow \infty$ the coefficient $D$ in (4.6) must be set equal to zero. Furthermore, as 
$Q_{l}\left(\mu^{*}\right)$ has a singularity at $\mu^{*}=1$, we set $d_{l}=0$ in to obtain a physically realistic solution. Hence we obtain

$$
F_{0}=C \frac{x^{-n}}{T} \exp \left(-\frac{x^{2}+x_{0}^{2}}{4 T}\right) \sum_{l=0}^{\infty} I_{\zeta}\left(\frac{x x_{0}}{2 T}\right) c_{l} P_{l}\left(\mu^{*}\right),
$$

as the form of the solution with $x$ given by Equation (2.3) with $b \neq 1$.

The coefficients $c_{l}$ in the solution (4.8) are determined by the condition

$$
F_{0} \rightarrow \frac{3 N_{s} \delta\left(x-x_{0}\right) \delta\left(\mu-\mu_{0}\right) \delta\left(\phi-\phi_{0}\right)}{8 \pi V p_{0}^{3} r_{0}^{2}|n+1|} \text { as } T \rightarrow 0\left(p \rightarrow p_{0}\right)
$$

implicit in equations (2.5). Noting that

$$
\delta\left(\mu-\mu_{0}\right) \delta\left(\phi-\phi_{0}\right)=\frac{\delta\left(\mu^{*}-1\right)}{2 \pi}=\sum_{l=0}^{\infty} c_{l} P_{l}\left(\mu^{*}\right),
$$

and using the orthogonality relations

$$
\int_{-1}^{1} P_{l}(x) P_{m}(x) d x=\frac{2}{2 l+1} \delta_{l, m}
$$

(Sneddon [20], p. 60), we find

$$
c_{l}=(2 l+1) P_{l}(1) / 4 \pi=(2 l+1) / 4 \pi .
$$

Finally by normalizing the solution according to (4.9) we obtain the monoenergetic point-source solution

$$
F_{0}(r, \theta, \phi, p)=\frac{3 N_{s} x_{0}^{2}}{16 \pi V p_{0}^{3} r_{0}^{2}|n+1|}\left(\frac{x_{0}}{x}\right)^{n} \frac{1}{T} \exp \left(-\frac{x^{2}+x_{0}^{2}}{4 T}\right) \sum_{l=0}^{\infty} \frac{2 l+1}{4 \pi} P_{l}\left(\mu^{*}\right) I_{b}\left(\frac{x x_{0}}{2 T}\right),
$$

where

$$
\begin{gathered}
x=\frac{2}{(1-b)}\left(r p^{3 / 2}\right)^{(1-b) / 2}, \quad T=\frac{3}{2 V} \int_{p}^{p_{0}} \kappa_{0}(z) z^{(1-3 b) / 2} d z, \\
\mu^{*}=\cos \Theta=\cos \theta \cos \theta_{0}+\sin \theta \sin \theta_{0} \cos \left(\phi-\phi_{0}\right), \\
\zeta=\left[n^{2}+e l(l+1)(n+1)^{2}\right]^{1 / 2}, \quad n=(b+1) /(1-b), \quad b \neq 1 .
\end{gathered}
$$

The spherically symmetric component of (4.13) is the monoenergetic-source solution of Webb and Gleeson [25] and of Toptygin [21] (that is, the term with $l=0)$. It is

$$
F_{0}=\frac{3 N x_{0}^{2}}{64 \pi^{2} V p_{0}^{3} r_{0}^{2}|n+1|}\left(\frac{x_{0}}{x}\right)^{n} \frac{1}{T} \exp \left(-\frac{x^{2}+x_{0}^{2}}{4 T}\right) I_{m}\left(\frac{x x_{0}}{2 T}\right)
$$

where the effective radial diffusion coefficient is $\kappa=\kappa_{0}(p) r^{b}, b \neq 1 ; 4 \pi r_{0}^{2} N$ is the injection rate; and $m=|n|$. 
This solution is also obtained by specifying a uniform distribution of point sources over a spherical shell at radius $r_{0}$, putting $N_{s}=N / 4 \pi$ in the point-source solution (4.13) and integrating over the solid angle element $d \Omega=2 \pi \sin \Theta d \Theta$ from $\Theta=0$ to $\Theta=\pi$.

Case (ii) $\kappa_{1}=\kappa_{0}(p) r, \kappa_{1} / \kappa_{\mathrm{n}}=e$.

The point-source solution for this case can be obtained in a completely analogous way to Case (i). The appropriate similarity solution form has been given in Webb ([24], 6.12), namely

$$
\begin{array}{r}
F_{0}=\frac{c}{\sqrt{\left(t-t_{s}\right)}} \exp \left[-\frac{x^{2}}{4\left(t-t_{s}\right)}\right. \\
\left.+\frac{\left(t-t_{s}+a\right) x}{\left(t-t_{s}\right)}-\frac{a^{2}}{t-t_{s}}-t+t_{s}\right] \\
\times \sum_{l=0}^{\infty} c_{l} P_{l}\left(\mu^{*}\right) \exp \left[-e l(l+1)\left(t-t_{s}\right)\right],
\end{array}
$$

where

$$
a=k / \gamma+t_{s}
$$

and the parameters $k, \gamma$ and $t_{s}$ are those of (3.8).

The point-source solution is obtained by substituting the parameter values (3.10) into the similarity form (4.16), using the values (4.12) for $c_{l}$, and appropriately normalizing the solution according to the source strength. It is

$$
\begin{aligned}
& F_{0}(r, \theta, \phi, p)=\frac{3 N_{8}}{16 \pi^{3 / 2} V p_{0}^{3} r_{0}^{2}} \frac{1}{\sqrt{(T)}} \exp \left[x-x_{0}-T-\frac{\left(x-x_{0}\right)^{2}}{4 T}\right] \\
& \times \sum_{l=0}^{\infty} \frac{2 l+1}{4 \pi} P_{l}\left(\mu^{*}\right) \exp [-e l(l+1) T],
\end{aligned}
$$

where

$$
x=-\frac{1}{2} \ln \left(2 r^{2} p^{3}\right), \quad T=\frac{3}{2 V} \int_{p}^{p_{0}} \kappa_{0}(z) z^{-1} d z .
$$

The spherically-symmetric component of the solution (4.18) gives the sphericallysymmetric monoenergetic source solution of Webb and Gleeson [25] and of Toptygin [21]. It is

$$
F_{0}=\frac{3 N}{64 \pi^{5 / 2} V P_{0}^{3} r_{0}^{2}} \frac{1}{\sqrt{T}} \exp \left[x-x_{0}-T-\frac{\left(x-x_{0}\right)^{2}}{4 T}\right],
$$

where the variables $x, T$ are those of equation (4.19) and $4 \pi r_{0}^{2} N$ is the injection rate. The solution (4.20) can be obtained by group methods, by a Laplace transform technique, or from a superposition of point source solutions with sources uniformly distributed over a spherical shell at radius $r_{0}$. Compare with equation (4.15) and its following remarks. 


\section{Concluding remarks}

The main aim of this paper has been to derive a monoenergetic point-source solution of the steady-state cosmic-ray equation of transport for a sphericallysymmetric model with anisotropic diffusion. The solution is a natural extension of the spherically-symmetric monoenergetic-source or Green's function solution of Webb and Gleeson [25] and of Toptygin [21]. The solution is of two forms, (4.13) or (4.18), according as the diffusion tensor is of the form (i) $\kappa_{\mathbb{b}}=\kappa_{0}(p) r^{b}, b \neq 1$, $\kappa_{\perp} / \kappa_{\|}=e$, or (ii) $\kappa_{\mathrm{q}}=\kappa_{0}(p) r, \kappa_{\perp} / \kappa_{\mathrm{q}}=e$.

The corresponding spherically-symmetric monoenergetic-source solution forms are (4.15) or (4.20), and are for a model with an effective radial diffusion coefficient $\kappa=\kappa_{0}(p) r^{b}$, and we note the results are independent of the transverse diffusion parameter $e$.

The monoenergetic-source solutions are very useful in displaying the physics of cosmic-ray propagation processes. Solutions in which more complex source or boundary conditions are specified (for example, continuous distributions at the boundary) do not show the physics so clearly since the resultant redistribution of particles in momentum and position is the combined result of the boundary and source conditions.

Finally, it is noted that the spherically-symmetric Green's function (4.15) has been used together with an appropriate Green's formula by Webb and Gleeson $[26,27]$ to construct solutions in which the momentum distribution $F_{0}$ is specified at infinity.

\section{REFERENCES}

[1] M. Abramowitz and I. A. Stegun, Handbook of mathematical functions (Dover, New York, 1965).

[2] G. W. Bluman, "Applications of the general similarity solution of the heat equation to boundary value problems", Quart. J. Appl. Math. 31 (1974), 403.

[3] G. W. Bluman and J. D. Cole, "The general similarity solution of the heat equation", J. Math. and Mechanics 18 (1969), 1025.

[4] G. W. Bluman and J. D. Cole, Similarity methods for differential equations (A.M.S. publications 13) (Springer-Verlag, New York, 1974).

[5] A. Z. Dolginov and I. N. Toptygin, "Diffusion of cosmic particles in the interplanetary medium", Geomag. and Aeron. 7 (1967), 785.

[6] A. Z. Dolginov and I. N. Toptygin, "Cosmic-rays in the interplanetary magnetic fields", Icarus 8 (1968), 54.

[7] L. A. Fisk, "Behaviour of cosmic-rays in the interplanetary medium" (Ph.D. Thesis, University of California, San Diego, 1969).

[8] L. A. Fisk and W. I. Axford, "Solar modulation of galactic cosmic-rays, 1", J. Geophys. Res. 74 (1969), 4973.

[9] L. J. Gleeson and W. I. Axford, "Cosmic-rays in the interplanetary medium", Astrophys. J. Lett. 149 (1967), L115.

[10] L. J. Gleeson and W. I. Axford, "The solar radial gradient of galactic cosmic-rays", Canad. J. of Physics 46 (1968), S937. 
[11] L. J. Gleeson and W. I. Axford, "Solar modulation of galactic cosmic-rays", Astrophys. J. 154 (1968), 1011.

[12] L. J. Gleeson and I. H. Urch, "A study of the force-field equation for the propagation of galactic cosmic-rays", Astrophys. and Space Sci. 25 (1973), 387.

[13] L. J. Gleeson and G. M. Webb, "Modulation and spectral redistribution of galactic cosmic-rays", Proc. 14th Int. Conf. on Cosmic-rays, Munich 3 (1975), 893.

[14] B. K. Harrison and F. B. Estabrook, "Geometric approach to invariance groups and solution of partial differential systems", J. Math. Phys. 12 (1971), 653.

[15] J. R. Jokipii and E. N. Parker, "On the convection, diffusion and adiabatic deceleration of cosmic-rays in the soiar wind", Astrophys. J. 172 (1970), 319.

[16] S. Lie, "Uber die Integration durch bestimmte Integrale von einer Klasse linearer partieller Differentialgleichungen", Arch. Math. VI (1881), 328.

[17] L. V. Ovsjannikov, Gruppovye svoystva differentsialny uravneni, Novosibirsk (1962) ("Group properties of differential equations", translated by G. W. Bluman, 1967).

[18] E. N. Parker, "The passage of energetic charged particles through interplanetary space" Planet. Space. Sci. 13 (1965), 9.

[19] E. N. Parker, "The effect of adiabatic deceleration on the cosmic-ray spectrum in the solar system", Planet. Space Sci. 14 (1966), 371.

[20] I. N. Sneddon, Special functions of mathematical physics and chemistry (Oliver and Boyd, Edinburgh, 1961).

[21] I. N. Toptygin, "Direct and inverse problem of cosmic-ray propagation in interplanetary space", Geomag. and Aeron. 13 (1973), 181.

[22] I. H. Urch, "Theoretical studies of the interplanetary medium" (Ph.D. Thesis, University of Adelaide, 1971).

[23] G. M., Webb, "Steady-state cosmic-ray propagation in interplanetary space" (Ph.D. Thesis, University of Tasmania, Hobart, 1976).

[24] G. M. Webb, "Similarity solutions of the steady-state cosmic-ray equation of transport", J. Aust. Math. Soc., Series B 19 (1976), 432.

[25] G. M. Webb and L. J. Gleeson, "Monoenergetic-source solutions of the steady-state cosmic-ray equation of transport", Proc. 13th Int. Conf. on Cosmic-rays, Denver University of Denver, Conf. Papers, 5, 1973, 3253).

[26] G. M. Webb and L. J. Gleeson, "Green's theorem and Green's functions for the steadystate cosmic-ray equation of transport", Astrophys. and Space Sci. 50 (1977), 205.

[27] G. M. Webb and L. J. Gleeson, "Green's formula for the cosmic-ray equation of transport", Proc. 15th Int. Conf. on Cosmic-Rays, Bulgaria, 3 (1977), 12.

\author{
Monash University \\ Clayton \\ Victoria 3168
}

